\title{
A Model for the Oxidation of Carbon Silicon Carbide
}

\section{Composite Structures}

\author{
Roy $M$. Sullivan ${ }^{*}$ \\ Structures Division \\ NASA Glenn Research Center \\ Mail Stop 49-7 \\ 21000 Brookpark Road \\ Brookpark, OH 44135
}

\section{ABSTRACT}

A mathematical theory and an accompanying numerical scheme have been developed for predicting the oxidation behavior of carbon silicon carbide $(\mathrm{C} / \mathrm{SiC})$ composite structures. The theory is derived from the mechanics of the flow of ideal gases through a porous solid. The result of the theoretical formulation is a set of two coupled nonlinear differential equations written in terms of the oxidant and oxide partial pressures. The differential equations are solved simultaneously to obtain the partial vapor pressures of the oxidant and oxides as a function of the spatial location and time. The local rate of carbon oxidation is determined using the map of the local oxidant partial

\footnotetext{
* Roy.sullivan@grc.nasa.gov fax no. (216)433-8300
} 
vapor pressure along with the Arrhenius rate equation. The nonlinear differential equations are cast into matrix equations by applying the Bubnov-Galerkin weighted residual method, allowing for the solution of the differential equations numerically. The numerical method is demonstrated by utilizing the method to model the carbon oxidation and weight loss behavior of $\mathrm{C} / \mathrm{SiC}$ specimens during thermogravimetric experiments. The numerical method is used to study the physics of carbon oxidation in carbon silicon carbide composites.

Keywords: A. Carbon fibers; B. Oxidation; C. Modeling; D. Diffusion, Porosity;

\section{INTRODUCTION}

The ability of carbon fiber-reinforced silicon carbide composites $(\mathrm{C} / \mathrm{SiC})$ to maintain its strength and stiffness at high temperatures as well as its low density make it an attractive candidate for many applications in future spacecraft. These applications include turbomachinery components and thrust chambers in future propulsion systems as well as control surfaces, leading edges and thermal protection systems for vehicle airframes. One of the more formidable obstacles to the widespread use of $\mathrm{C} / \mathrm{SiC}$ structures in future launch vehicles is that the carbon fibers oxidize at medium to high temperatures in an environment in which oxygen is present. This does not forbid the use of $\mathrm{C} / \mathrm{SiC}$ in future launch vehicle applications, as long as it can be verified through testing and analysis that the component will maintain its strength and stiffness throughout its service life, with the demonstration of sufficient safety factors. As such, an assessment 
of the oxidation behavior of $\mathrm{C} / \mathrm{SiC}$ composite structures must be included along with the usual design analysis activities such as the thermal, dynamic and thermostructural analysis of the component. It is therefore necessary to develop a tool that is capable of determining the spatial distribution of the extent of oxidation and the residual strength and stiffness in the $\mathrm{C} / \mathrm{SiC}$ component as a function of the time, temperature and environmental oxygen concentrations to which the $\mathrm{C} / \mathrm{SiC}$ structure is exposed. Currently, no such oxidation analysis tool is available to designers, who wish to utilize $\mathrm{C} / \mathrm{SiC}$ composites.

Oxygen attacks the carbon in $\mathrm{C} / \mathrm{SiC}$ composites both on the surface and in the interior of the composite. The oxygen achieves access to the interior of the composite via an interconnected network of passageways, which are formed by the combination of matrix cracks and void spaces, which exist both within the fiber bundles as well as between adjacent plies. Oxygen may also flow through separations of the fiber/coating and coating/matrix interfaces. The matrix cracks and interfacial separations are due to tensile stresses, which are a result of the thermal expansion mismatch between the carbon fibers and the silicon carbide matrix in concert with the temperature excursions during processing and cool down [1]. The large free spaces between plies and the void spaces within the fiber bundles are both due to insufficient void filling during matrix infiltration. The void spaces and matrix cracks are illustrated in Fig. 1 where the microstructure of a 2-D $\mathrm{C} / \mathrm{SiC}$ composite is shown. The oxidation of carbon in the interior of $\mathrm{C} / \mathrm{SiC}$ composites is strictly tied to the transport of oxygen into and the transport of oxides out of the material. Any viable oxidation model for $\mathrm{C} / \mathrm{SiC}$ composites must include the 
solution of species transport equations as the transport has a direct impact on the rate of carbon oxidation.

Oxidation models have been developed in the past in order to study the physics of the oxidation process in carbon fiber-reinforced composites. Medford [2] proposed a model to predict the oxidation of the Space Shuttle's carbon-carbon wing leading edge, by simulating the diffusion of oxygen to the carbon-carbon substrate down a fissure in the SiC coating. Eckel, et al. [3] proposed a similar model to determine the oxidation recession rate of a single carbon fiber embedded in a non-reactive matrix. Halbig [4] adapted Eckel, et al.'s model to simulate the fiber surface recession in $\mathrm{C} / \mathrm{SiC}$ specimens. His approach presupposes a crack extending through the specimen gage section, bridged by an array of continuous carbon fibers. All three models assume steady state diffusion of the oxidant to the site of oxidation.

Although these previous studies have provided insight into the physics of carbon oxidation in ceramic composites, these approaches are not readily applicable to support the design analysis of $\mathrm{C} / \mathrm{SiC}$ structures as they are impractical for predicting the residual strength and stiffness as a function of space and time for any arbitrarily-shaped $\mathrm{C} / \mathrm{SiC}$ structure. Indeed, these previous methods study the problem of carbon oxidation on a very fine scale, retaining the heterogeneous nature of the composite. As a result, these methods would be too cumbersome if they were applied to analyze $\mathrm{C} / \mathrm{SiC}$ structures on a global level. For this purpose, a numerical model that treats the $\mathrm{C} / \mathrm{SiC}$ composite as a continuum is needed. 
The purpose of this paper is to describe the development of an accurate analysis method that simulates the oxidation behavior of $\mathrm{C} / \mathrm{SiC}$ composite structures in high temperature applications. In this paper, the mathematical foundation of the method is presented. The theory is derived by assuming that the $\mathrm{C} / \mathrm{SiC}$ material is a homogeneous, orthotropic porous body with a solid skeleton that is a mixture of multiple solid constituents where some of the constituents are reactive. The oxygen (oxidant) and the oxides (product) flow through the pore network and the partial pressures of the gases vary with space and time. The pore volume of this porous body represents the collective volumes of the matrix cracks, the fiber/coating and coating/matrix interfacial separations, and both types of void volumes (inter-ply and intra-bundle). Applying the fundamentals of porous media to this problem, namely the mass conservation equation for each gas specie as well as the transport mechanisms, a set of coupled, nonlinear differential equations is obtained. The solution of these differential equations yields the partial vapor pressures of the oxidant and oxides as a function of space and time. The local rate of carbon oxidation is determined as a function of space and time using the map of the local oxidant partial vapor pressure along with the Arrhenius rate equation. The BubnovGalerkin weighted residual method is used to cast the governing differential equations into matrix equations in order to perform the solution of the differential equations numerically.

In the application section of this paper, we demonstrate the use of the numerical method by applying it to simulate the carbon oxidation and weight loss behavior of $\mathrm{C} / \mathrm{SiC}$ 
specimens during thermogravimetric analysis (TGA) experiments. Using the numerical method, we deduce the value of two key material parameters as well as the variation of these parameters with temperature and we demonstrate the profound influence of temperature on the oxidation behavior.

\section{THEORETICAL FORMULATION}

\subsection{Mass Continuity for Flow Through Porous Media}

In order to develop the theory to model the oxidation process, we make use of the basic principals of porous media theory, namely the equation for the continuity of mass of gaseous species flowing through a porous solid as well as mechanisms for transport in the porous solid. It is assumed that the oxygen and the products of the oxidation reaction (oxides) exist only in the pores of the material in a gas form and that these gases behave ideally, that the solid skeleton consists of a mixture of both silicon carbide and carbon in the solid form and that the carbon is oxidized at the interface between the solid skeleton and the pore, in other words, at the wall of the pore space.

The local form of the mass continuity equations for oxygen and the oxide species, flowing through a porous solid body, may be written as

$$
\phi \frac{\partial \rho_{o x}^{p}}{\partial t}+\nabla \cdot J_{o x}=\lambda_{1} \frac{M_{o x}}{M_{c}}\left(\Re_{c f}+\Re_{p c}\right)
$$


and

$$
\phi \frac{\partial \rho_{c o}^{p}}{\partial t}+\nabla \cdot J_{c o}=-\lambda_{2} \frac{M_{c o}}{M_{c}}\left(\Re_{c f}+\Re_{p c}\right)
$$

respectively, where $\phi$ is the volumetric porosity and $\nabla$ is the gradient operator and where $\rho_{i}^{p}$ is the local partial density in the pore volume, $M_{i}$ is the molecular weight and $J_{i}$ is the local mass flux vector for specie $i$. The subscripts $o x$, $c o$ and $c$ refer to the oxygen, the oxide (either carbon monoxide or carbon dioxide) and the carbon (solid form) species, respectively.

In equations (1a) and (1b), the symbols $\Re_{c f}$ and $\Re_{p c}$ denote the local time rate of carbon fiber mass and pyrocarbon coating mass consumption due to the oxidation reaction per unit bulk volume, respectively. Further, $\lambda_{1}$ and $\lambda_{2}$ are the stoichiometric constants for the oxidation reactions. That is, $\lambda_{1}$ is the ratio of the number of moles of oxygen consumed in the oxidation reaction to the number of moles of carbon consumed in the reaction and $\lambda_{2}$ is the ratio of the number of moles of oxide produced in the oxidation reaction to the number of moles of carbon consumed. As such, the right hand side (RHS) of (1a) is the local rate of oxygen mass consumed in the oxidation reaction per unit bulk volume and the RHS of (1b) is the local rate of oxide mass produced by the oxidation reaction per unit bulk volume. 
Upon substituting the ideal gas law for each specie $i\left(\rho_{i}^{p}=p_{i} M_{i} / R T\right)$ into the first term in equations (1a) and (1b) and upon performing the differentiation, we obtain, after rearranging,

$$
\phi \frac{M_{o x}}{R T} \frac{\partial p_{o x}}{\partial t}+\nabla \cdot J_{o x}-\lambda_{1} \frac{M_{o x}}{M_{c}}\left(\Re_{c f}+\Re_{p c}\right)-\phi \frac{\rho_{o x}^{p}}{T} \frac{d T}{d t}=0
$$

and

$$
\phi \frac{M_{c o}}{R T} \frac{\partial p_{c o}}{\partial t}+\nabla \cdot J_{c o}+\lambda_{2} \frac{M_{c o}}{M_{c}}\left(\Re_{c f}+\Re_{p c}\right)-\phi \frac{\rho_{c o}^{p}}{T} \frac{d T}{d t}=0
$$

\subsection{Mass Flux Constitutive Relations}

We will allow for the diffusion of oxygen and the oxides through the pore network via two mechanisms: gas pressure gradient-driven flow and concentration gradient-driven flow. Thus, we may write the mass flux vector of specie $i$ as the sum of two mass flux vectors as

$$
J_{i}=J_{i}^{\alpha}+J_{i}^{\beta}
$$


where $J_{i}^{\alpha}$ is the mass flux associated with the average velocity of the gas mixture and is gas pressure gradient-driven and where $J_{i}^{\beta}$ is the flux of specie $i$ relative to the mixture average velocity and is concentration gradient-driven.

The expression for the gas pressure gradient-driven flow is attributed to Darcy [5] and is written as

$$
\boldsymbol{J}_{i}^{\alpha}=-\rho_{i}^{p} \frac{1}{\mu_{g}} \mathbf{k} \cdot \nabla p
$$

where $\mathbf{k}$ is the second-order material permeability tensor, $\mu_{g}$ is the viscosity of the gas mixture and $p$ is the total gas pressure.

The concentration gradient-driven flow is given by modifying Fick's law [6] as

$$
J_{i}^{\beta}=-\rho^{p} D_{A B} \varphi^{A} \cdot \nabla\left(\frac{\rho_{i}^{p}}{\rho^{p}}\right)
$$

where $\rho^{p}$ is the local gas mixture density in the pore and $D_{A B}$ is the diffusivity of gas specie A with respect to gas specie $\mathrm{B}$, and $\varphi^{A}$ is the second-order areal porosity tensor. The diffusivity $D_{A B}$ is given by the Chapman-Enskog equation which is derived from the kinetic theory of gases [6]. 
The areal porosity tensor is a measure of the resistance to concentration gradientdriven flow through the pore network. We note that Bacos, et al. [7] use the ratio $\varepsilon / \tau$ to represent this resistance, where the quantity $\varepsilon$ denotes the porosity and $\tau$ is defined as a tortuosity factor. In the present study, we will use the areal porosity tensor to represent the ratio $\varepsilon / \tau$. As such, $\varphi^{A}$ represents the combined resistance to concentration gradient-driven diffusion due to both the tortuosity and the fraction of the cross-sectional area which is occupied by pore volume. The value of the areal porosity will, of course, depend on the pore morphology.

Making use of the ideal gas law for each specie $i\left(\rho_{i}^{p}=p_{i} M_{i} / R T\right)$ as well as the ideal gas law for the mixture ( $\rho^{p}=p M_{g} / R T$ ), we can rewrite equation (5) in terms of the partial pressures $p_{i}$ :

$$
\boldsymbol{J}_{i}^{\beta}=-\rho^{p} D_{A B} \frac{M_{i}}{M_{g}} \boldsymbol{\varphi}^{A} \cdot \nabla\left(\frac{p_{i}}{p}\right),
$$

where $M_{g}$ is the molecular weight of the gas mixture.

Substituting equations (4) and (6) into (3) leads to 


$$
\begin{aligned}
\boldsymbol{J}_{o x}=-\rho_{o x}^{p} & \frac{1}{\mu_{g}} \mathbf{k} \cdot \nabla p_{o x}-D_{A B} \frac{M_{o x}}{R T} \frac{p_{c o}}{p} \boldsymbol{\varphi}^{A} \cdot \nabla p_{o x} \\
& -\rho_{o x}^{p} \frac{1}{\mu_{g}} \mathbf{k} \cdot \nabla p_{c o}+D_{A B} \frac{M_{o x}}{R T} \frac{p_{o x}}{p} \boldsymbol{\varphi}^{A} \cdot \nabla p_{c o}
\end{aligned}
$$

and

$$
\begin{aligned}
\boldsymbol{J}_{c o}=-\rho_{c o}^{p} & \frac{1}{\mu_{g}} \mathbf{k} \cdot \nabla p_{c o}-D_{A B} \frac{M_{c o}}{R T} \frac{p_{o x}}{p} \boldsymbol{\varphi}^{A} \cdot \nabla p_{c o} \\
& -\rho_{c o}^{p} \frac{1}{\mu_{g}} \mathbf{k} \cdot \nabla p_{o x}+D_{A B} \frac{M_{c o}}{R T} \frac{p_{c o}}{p} \boldsymbol{\varphi}^{A} \cdot \nabla p_{o x}
\end{aligned}
$$

In the derivation of equations (7a) and (7b), it was necessary to employ Dalton's law for the gas mixture $\left(p=p_{o x}+p_{c o}\right)$, the distributive property of the gradient operator $\left(\nabla p=\nabla p_{o x}+\nabla p_{c o}\right)$ and the ideal gas law for the gas mixture.

It is apparent that, upon substituting equations (7a) and (7b) into (2a) and (2b), we will arrive at two coupled nonlinear differential equations written in terms of the partial pressures. These equations will be coupled, since terms involving the gradients $\nabla p_{o x}$ and $\nabla p_{c o}$ will appear in both equations. These equations will be nonlinear, since (7a) and (7b) contain terms that involve the product of the partial densities and the gradients of the partial pressures as well as terms that involve the product of the partial pressures and the gradients of the partial pressures. The two nonlinear differential equations must be solved simultaneously at each time step to obtain the partial pressures at each spatial location. In 
the following sections, a numerical approach is presented to perform the simultaneous solution of these equations.

\subsection{Determination of Oxidation Reaction Rates}

The time rate of the carbon oxidation reaction is a function of the absolute temperature and the vapor pressure of the oxidant [8]. The dependence of the reaction rate on temperature and pressure is given by the Arrhenius rate equation. As we are concerned with a solid mixture containing carbon, then for any unit volume of material, we can write the Arrhenius equation in terms of a density ratio as

$$
\frac{d}{d t}\left(\frac{\rho_{c}}{\rho_{c}^{o}}\right)_{\text {oxidation }}=-k_{o} \exp \left(\frac{-E_{a}}{R T}\right) p_{o x}^{n}
$$

where $\rho_{c}$ and $\rho_{c}^{o}$ are the instantaneous mass density and initial mass density of carbon in the solid mixture, respectively, and where $k_{o}$ is the pre-exponential coefficient, $E_{a}$ is the activation energy of the oxidation reaction and $n$ is the order of the reaction. The Arrhenius constants $k_{o}, E_{a}$ and $n$ are obtained by curve fitting thermogravimetric analysis (TGA) measurements to the Arrhenius equation.

In applying equation (8), it is necessary to recognize a few key differences between the oxidation of carbon fibers or pyrocarbon coating in TGA tests and the oxidation of these constituents in $\mathrm{C} / \mathrm{SiC}$ composites. First, the ambient partial pressure of 
oxygen in the TGA experiments is constant and specified as a test condition, whereas the oxygen partial pressure in the $\mathrm{C} / \mathrm{SiC}$ composite varies with time and spatial position. Second, the carbon surface area that is exposed to oxygen vapor may be approximated as close to $100 \%$ in the TGA experiments, whereas this surface area fraction in the $\mathrm{C} / \mathrm{SiC}$ composite is considerably less than this estimate. It is therefore necessary to modify equation (8) in order to use the Arrhenius rate equation to determine the local rate of oxidation in $\mathrm{C} / \mathrm{SiC}$ composites.

We note that by definition, $\Re_{c f} \equiv\left(d \rho_{c f} / d t\right)_{\text {oxidation }}$ and $\Re_{p c} \equiv\left(d \rho_{p c} / d t\right)_{\text {oxidation }}$, where $\rho_{c f}$ and $\rho_{p c}$ are the local mass density of carbon fiber and local mass density of pyrocarbon coating, respectively. The local mass densities and the local volume fractions are related by $\rho_{c f}=\widetilde{\rho}_{c} v_{c f}$ and $\rho_{p c}=\widetilde{\rho}_{c} v_{p c}$, where $v_{c f}$ and $v_{p c}$ are the volume fraction of carbon fiber and pyrocarbon coating, respectively and where $\widetilde{\rho}_{c}$ is the intrinsic density of carbon.

Given these considerations, equation (8) may be rewritten separately for both the carbon fibers and pyrocarbon coating, as,

$$
\Re_{c f}=-\widetilde{\rho}_{c} v_{c f}^{o}\left(\frac{p_{o x}}{p_{o x}^{*}}\right)^{n_{c f}} \psi^{c f} k_{o}^{c f} \exp \left(\frac{-E_{a}^{c f}}{R T}\right)
$$

and 


$$
\Re_{p c}=-\widetilde{\rho}_{c} v_{p c}^{o}\left(\frac{p_{o x}}{p_{o x}^{*}}\right)^{n} p c \psi^{p c} k_{o}^{p c} \exp \left(\frac{-E_{a}^{p c}}{R T}\right),
$$

where $v_{c f}^{o}$ and $v_{p c}^{o}$ are the initial values of the volume fractions and where $k_{o}^{c f}, E_{a}^{c f}$ and $n_{c f}$ are the Arrhenius constants associated with the carbon fiber oxidation and $k_{o}^{p c}$, $E_{a}^{p c}$ and $n_{p c}$ are the Arrhenius constants associated with the pyrocarbon coating oxidation. The quantity $p_{o x}^{*}$ is the ambient oxygen vapor pressure in the TGA experiments in which the values of the Arrhenius constants were determined. Furthermore, the quantities $\psi^{c f}$ and $\psi^{p c}$ have been introduced as the fraction of the fiber surface area and fraction of pyrocarbon coating surface area that is exposed to oxygen in the pore volume. We refer to these quantities as the exposed surface area fractions. The value of these parameters will also depend upon the pore morphology.

\section{FINITE ELEMENT FORMULATION}

Applying the Bubnov-Galerkin method [9], the finite element form of (2a) and (2b) become

$$
\int_{D^{e}} N_{i} \phi \frac{M_{o x}}{R T} N_{j} \frac{\partial p_{o x}}{\partial t} d D^{e}+\int_{D^{e}} N_{i} \nabla \cdot J_{o x} d D^{e}
$$




$$
-\int_{D^{e}} N_{i} \lambda_{1} \frac{M_{o x}}{M_{c}} N_{j}\left(\Re_{c f j}+\Re_{p c j}\right) d D^{e}-\int_{D^{e}} N_{i} \phi \frac{\rho_{o x}^{p}}{T} \frac{d T}{d t} d D^{e}=0
$$

and

$$
\begin{aligned}
& \int_{D^{e}} N_{i} \phi \frac{M_{c o}}{R T} N_{j} \frac{\partial p_{c o} j}{\partial t} d D^{e}+\int_{D^{e}} N_{i} \nabla \cdot J_{c o} d D^{e} \\
& \quad+\int_{D^{e}} N_{i} \lambda_{2} \frac{M_{c o}}{M_{c}} N_{j}\left(\Re_{c f j}+\Re_{p c j}\right) d D^{e}-\int_{D^{e}} N_{i} \phi \frac{\rho_{c o}^{p}}{T} \frac{d T}{d t} d D^{e}=0
\end{aligned}
$$

where $N_{i}$ are the element shape functions and $D^{e}$ is the domain of each element and where $p_{o x_{i}}, p_{c o i}, \Re_{c f i}$ and $\Re_{p c_{i}}$ are the element nodal values of $p_{o x}, p_{c o}, \Re_{c f}$ and $\Re_{p c}$, respectively.

Using the Product Rule of differentiation along with Gauss' Theorem [10] and upon substituting equations (7a) and (7b), the second term in equations (10a) and (10b) can be expanded as

$$
\int_{D^{e}} N_{i} \nabla \cdot \boldsymbol{J}_{o x} d D^{e}=\oint_{\Gamma^{e}} N_{i} \boldsymbol{J}_{o x} \cdot \boldsymbol{n} d \Gamma^{e}+\int_{D^{e}} \nabla N_{i} \cdot \rho_{o x}^{p} \frac{1}{\mu_{g}} \mathbf{k} \cdot \nabla p_{o x} d D^{e}
$$




$$
\begin{gathered}
+\int_{D^{e}} \nabla N_{i} \cdot D_{A B} \frac{M_{o x}}{R T}\left(\frac{p_{c o}}{p}\right) \varphi^{A} \cdot \nabla p_{o x} d D^{e}+\int_{D^{e}} \nabla N_{i} \cdot \rho_{o x}^{p} \frac{1}{\mu_{g}} \mathbf{k} \cdot \nabla p_{c o} d D^{e} \\
-\int_{D^{e}} \nabla N_{i} \cdot D_{A B} \frac{M_{o x}}{R T}\left(\frac{p_{o x}}{p}\right) \varphi^{A} \cdot \nabla p_{c o} d D^{e}
\end{gathered}
$$

and

$$
\begin{gathered}
\int_{D^{e}} N_{i} \nabla \cdot J_{c o} d D^{e}=\oint_{\Gamma^{e}} N_{i} J_{c o} \cdot n d \Gamma^{e}+\int_{D^{e}} \nabla N_{i} \cdot \rho_{c o}^{p} \frac{1}{\mu_{g}} \mathbf{k} \cdot \nabla p_{c o} d D^{e} \\
+\int_{D^{e}} \nabla N_{i} \cdot D_{A B} \frac{M_{c o}}{R T}\left(\frac{p_{o x}}{p}\right) \varphi^{A} \cdot \nabla p_{c o} d D^{e}+\int_{D^{e}} \nabla N_{i} \cdot \rho_{c o}^{p} \frac{1}{\mu_{g}} \mathbf{k} \cdot \nabla p_{o x} d D^{e} \\
-\int_{D^{e}} \nabla N_{i} \cdot D_{A B} \frac{M_{c o}}{R T}\left(\frac{p_{c o}}{p}\right) \varphi^{A} \cdot \nabla p_{o x} d D^{e}
\end{gathered}
$$

where $\Gamma^{e}$ is the boundary of the element and $n$ is the outward unit vector normal to the boundary. The boundary integral terms in equations (11a) and (11b) allow for the application of unconstrained boundary conditions. As indicated, the integration is performed in a closed path around the element boundary. The integral terms are only nonzero for elements where mass flux boundary conditions are imposed. 
In order to linearize equations (11a) and (11b), the partial pressures which are operated on by the gradient operator are treated as the solution variables and it is assumed that the partial densities and the partial pressures in brackets in (11a) and (11b) are constant within the element over the duration of any arbitrary time step. Therefore, the partial pressures in brackets and the partial densities may be taken outside the volume integral and the gradient terms $\nabla p_{o x}$ and $\nabla p_{c o}$ are replaced with the approximations $\nabla N_{i} p_{o x_{i}}$ and $\nabla N_{i} p_{c o i}$. Furthermore, it is assumed that the temperature, the gas viscosity, the diffusivity and the volumetric porosity are all constant within the element (although these quantities may vary from element to element) and therefore these quantities may also be taken outside the volume integrals in equations (11a) and (11b).

After substituting equations (11a) and (11b) into (10a) and (10b), and recognizing that the element nodal values of the partial pressures, $p_{o x_{i}}$ and $p_{c o_{i}}$, as well as the temporal derivatives, $\partial p_{o x_{i}} / \partial t$ and $\partial p_{c o_{i}} / \partial t$, are not functions of the spatial variables, equations (14a) and (14b) may be written in the matrix form

$$
\left[\begin{array}{cc}
C O X & 0 \\
0 & C C O
\end{array}\right] \frac{d}{d t}\left\{\begin{array}{l}
p_{o x_{j}} \\
p_{c o_{j}}
\end{array}\right\}+\left[\begin{array}{cc}
\boldsymbol{K} O \boldsymbol{X} & \boldsymbol{K X C} \\
\boldsymbol{K C X} & \boldsymbol{K C O}
\end{array}\right]\left\{\begin{array}{l}
p_{o x_{j}} \\
p_{c o_{j}}
\end{array}\right\}=\left\{\begin{array}{l}
\boldsymbol{F O X} \\
\boldsymbol{F C O}
\end{array}\right\}
$$

Expressions for the elements of the capacitance and stiffness matrices and the force vector in equation (12) are listed in the Appendix. In the expressions in the Appendix, the symbol $\left({ }^{\wedge}\right)$ above the quantity denotes the element average value of that quantity. 
Equation (12) can be represented more concisely as

$$
[\boldsymbol{C}]_{n} \frac{d}{d t}\{P\}_{n}+[\boldsymbol{K}]_{n}\{P\}_{n}=\{\boldsymbol{F}\}_{n}
$$

where $\{P\}$ represents the solution vector $\left\{p_{o x_{i}}, p_{c o_{i}}\right\}$ and where the subscript $n$ indicates the matrices and vectors at time $t_{n}$. Using the Backwards Difference Method [10], the time rate of change of the partial pressure vector is

$$
\frac{d\{P\}_{n}}{d t}=\frac{\{P\}_{n}-\{P\}_{n-1}}{\Delta t_{n}}
$$

where $\Delta t_{n}=t_{n}-t_{n-1}$. Substituting (14), equation (13) may be rewritten in the simple matrix form

$$
\left\langle\boldsymbol{K}_{\text {eff }}\right\rfloor_{n}\{P\}_{n}=\left\{\boldsymbol{F}_{\text {eff }}\right\}_{n}
$$

where

$$
\left[\boldsymbol{K}_{\text {eff }}\right]_{n}=\frac{1}{\Delta t_{n}}[\boldsymbol{C}]_{n}+[\boldsymbol{K}]_{n}
$$

and 


$$
\left\{\boldsymbol{F}_{\text {eff }}\right\}_{n}=\frac{1}{\Delta t_{n}}[\boldsymbol{C}]_{n}\{P\}_{n-1}+\{\boldsymbol{F}\}_{n}
$$

Thus, the partial pressures $p_{o x}$ and $p_{c o}$ at each node are determined by the

solution of equation (15) at each time step. The effective stiffness matrix $\left\lfloor\boldsymbol{K}_{\text {eff }}\right\rfloor_{n}$ and

effective force vector $\left\{F_{\text {eff }}\right\}_{n}$ for time $t_{n}$ are calculated using the expressions in the Appendix, equations (16a) and (16b) and the partial pressure values determined at the previous time step, $t_{n-1}$. A flow chart illustrating the steps involved in the numerical solution routine is shown in Fig. 2.

\section{APPLICATION OF THE NUMERICAL APPROACH: TGA SIMULATION}

\subsection{Experiment Description}

Halbig [4] measured the weight loss behavior of 2-D plain weave $\mathrm{C} / \mathrm{SiC}$ laminated composites. The material was fabricated by GE Power System Composites of Newark, DE. The material was fabricated with T300 carbon fibers and infiltrated with a SiC matrix through a chemical vapor infiltration (CVI) process. Specimens were machined into $2.54 \mathrm{~cm}$-long prismatic bars with a rectangular cross-section of $0.3175 \mathrm{~cm}$ $\mathrm{x} 1.27 \mathrm{~cm}$. The specimens were machined such that the through-thickness specimen direction $(0.3175 \mathrm{~cm})$ was normal to the fabric plane. The weight loss was measured in an environment of pure oxygen at $1 \mathrm{~atm}$ pressure. The residual weight was measured and 
recorded continuously with time. A schematic of the TGA experiment and a description of the experimental procedure are given in Opila [11]. TGA measurements were performed at a number of temperatures. The results of these measurements are shown in Fig. 3 where the residual weight fraction (ratio of instantaneous weight to initial weight) is plotted versus time for each temperature.

Fig. 3 illustrates the significant influence of temperature on the oxidation and weight loss behavior. Below $700{ }^{\circ} \mathrm{C}$, the rate of oxidation and weight loss increases with increasing temperature. Above $700{ }^{\circ} \mathrm{C}$, the rate of weight loss decreases with increasing temperature. In addition, at temperatures below $700{ }^{\circ} \mathrm{C}$, the weight loss profile has a sigmoidal shape. That is, initially, the oxidation and weight loss rates are low. The rate of oxidation increases with time and eventually reaches a constant rate at a percent weight loss of approximately $15 \%$.

It should be noted that the sigmoidal shape of the weight loss curves at the lower temperatures was also observed by Lamouroux, et al. [1] while performing TGA experiments on T300 carbon fibers coated with a pyrocarbon coating as well as by Halbig [12] while measuring the weight loss behavior of T300 fibers. As Ismail [13] suggests, the rise in oxidation rate within the initial portion of the weight loss curve is most likely associated with the development of porosity in the outer surface of the carbon fibers. As porosity increases, more active sites become available for oxygen, thereby increasing the rate of carbon oxidation. The influence of porosity development on the rate of oxidation 
is only observed in the initial portion of the weight loss curve, presumably when the outer portion of the fiber is being oxidized.

At temperatures of $700{ }^{\circ} \mathrm{C}$ and above, the weight loss curves no longer exhibit the sigmoidal shape. The weight loss profiles maintain a constant sign of curvature (concave up and right). This is the case in both the $\mathrm{C} / \mathrm{SiC}$ composite weight loss behavior shown in Fig. 3 and in the T300 weight loss behavior report by Halbig [12] and by Lamouroux, et al. [1]. Perhaps at the higher temperatures, the rate is no longer dependent upon the fiber porosity or perhaps, at the higher temperatures, the thermal expansion of the fibers results in sufficient porosity.

\subsection{Numerical Simulation Approach}

Two-dimensional, three-node triangular elements were chosen to implement the finite element method. The mesh used for the simulation of the TGA experiment, shown in Fig. 4, represents a cross-sectional slice of the TGA specimen. The boundary and initial conditions which were applied to the finite element mesh are also shown in Fig. 4.

The material constants used for the numerical simulation are listed in Table 1. In this numerical simulation, we will treat the carbon fibers and pyrocarbon coating as one

constituent. We therefore make the substitution $\psi^{c f}=\psi^{p c}=\psi$. Furthermore, we assume that the Arrhenius constants are the same for both constituents. The volumetric 
porosity was assumed to be on the order of $10 \%$ and an initial combined carbon volume fraction was assumed to be $50 \%$.

In the oxidation of carbon fibers and pyrocarbon coating in $\mathrm{C} / \mathrm{SiC}$ composites, multiple reaction mechanisms are possible (Refs. [3], [8], [14]). For the purpose of demonstrating the numerical method, we will concentrate our attention on the reaction $\mathrm{C}+\mathrm{O}_{2} \rightarrow \mathrm{CO}_{2}$. Thus, $\lambda_{1}=\lambda_{2}=1$.

The values of $k_{o}$ and $E_{a}$ in Table 1 were obtained by Halbig [12] by conducting weight loss measurements on T300 carbon fibers. The activation energy was calculated in the usual manner, by plotting the natural log of the rate of weight loss versus the inverse of the absolute temperature. The slope of this plot yields the value of the activation energy. The rate of weight loss was obtained from the slope of the weight loss curves at the midpoint of the curves. In this manner, the variation of the rate of carbon fiber weight loss with temperature is input into the numerical method.

As a result of this approach, however, the sigmoidal signature of the weight loss curves at the lower temperatures, that is evident in Fig. 3, will not be passed into the numerical solution method. Recall that the sigmoidal shape is caused by the timedependent formation of porosity in the carbon fibers in the initial portion of the weight loss curves. Since a model which accounts for fiber porosity development and the associated rise in carbon oxidation rate is not part of the present numerical approach, we 
can not expect the numerical solution to reproduce the sigmoidal shape of the weight loss curves at the lower temperature regime.

Although 2-D plain weave $\mathrm{C} / \mathrm{SiC}$ composites are orthotropic with regard to many material response properties, including the gas transport parameters of material permeability and areal porosity, we will assume, for the sake of simplicity, that the material is isotropic in regards to these transport parameters. Therefore, the permeability and areal porosity tensors take the form

$$
\mathbf{k}=\left[\begin{array}{ccc}
k & 0 & 0 \\
0 & k & 0 \\
0 & 0 & k
\end{array}\right] \quad \text { and } \quad \boldsymbol{\varphi}^{\mathbf{A}}=\left[\begin{array}{ccc}
\phi^{A} & 0 & 0 \\
0 & \phi^{A} & 0 \\
0 & 0 & \phi^{A}
\end{array}\right]
$$

where $k$ and $\phi^{A}$ are scalar quantities representing the material permeability and areal porosity, respectively.

Recognizing that the weight fraction is equivalent to the composite density fraction, the weight fraction remaining at each time step may be calculated with the numerical solution by: 1) calculating the volume average carbon volume fraction within the finite element mesh $\bar{v}_{c}$ and 2) employing the rule of mixture expression to calculate the instantaneous composite density, $\bar{\rho}=\widetilde{\rho}_{c} \bar{v}_{c}+\rho_{S i C} v_{S i C}$, where $\rho_{S i C}$ is the density of silicon carbide and $v_{S i C}$ is the volume fraction of the matrix. We will use this approach 
to calculate the weight fraction remaining at each time step, in order to compare the numerical results with the measured TGA response.

\section{NUMERICAL RESULTS}

In order to investigate the effect of material permeability on the oxidation behavior in the TGA experiment, a series of numerical simulations were performed using a wide range of permeability values. The results of these simulations revealed that the value of the material permeability had little or no effect on the oxidation behavior in the TGA experiment. The fact that material permeability has no influence on the oxidation behavior in the TGA experiment simulation indicates that the primary mode of oxygen diffusion in the TGA specimen is not Darcy flow (total gas pressure gradient-driven flow). This, of course, is somewhat expected, since under the conditions that have been imposed, the presence of significant total gas pressure gradients would not be expected. For all remaining numerical simulations, we will assume a value of $10^{-4} \mathrm{~m}^{2} / \mathrm{MPa}$-sec for the permeability-to-gas viscosity ratio.

As previously discussed, the pore structure of $\mathrm{C} / \mathrm{SiC}$ composites is largely attributed to the residual stresses in the $\mathrm{SiC}$ matrix that result from processing and these residual stresses result in matrix cracks and debonds between the carbon fibers, the pyrocarbon coating and the $\mathrm{SiC}$ matrix. This crack network in combination with the void volume constitutes the pore volume of the porous material. Since the magnitude of the 
residual stresses is a function of the temperature, namely the amount of departure from the processing temperature, the amount of cracking and crack opening will also depend upon the temperature. Likewise, one would expect that the crack openings and interfacial debond separations will close upon reheating, and that the amount of closure is a function of the temperature. We also recognize that as the areal porosity and the surface area fractions are dependent upon the pore morphology, the value of these two material parameters must be affected by crack opening and closing. Specifically, larger crack openings and interface separations would result in a larger value for the areal porosity due to a smaller value for the tortuosity and, to a lesser extent, a larger value for the volumetric porosity. Larger crack openings and interface separations would also result in a larger value for the surface area fraction, since more of the carbon surfaces would be accessible to oxygen. Given these considerations, it is logical to assume that the areal porosity and the surface area fraction will be a minimum at the processing temperature and that the value of these parameters will increase as the temperature decreases from the processing temperature.

Unfortunately, the values of these two parameters are not available for the $\mathrm{C} / \mathrm{SiC}$ material that is the subject of this study and, in fact, it may be impossible to measure the in-situ value of these parameters at elevated temperatures. For this reason, a series of numerical experiments were performed using the numerical method and, through trial and error, the variation of the areal porosity and the surface area fraction with temperature that yielded the best match with the measured oxidation behavior was determined. This was based on a comparison between the rates of oxidation predicted with the numerical 
method and the measured rates of oxidation at all TGA test temperatures. The variation of the areal porosity and surface area fraction with temperature that produced the best correlation between the measured and predicted rates of oxidation is shown in Fig. 5. We note that the variation of these two parameters with temperature shown in Fig. 5 is completely consistent with our intuition, given their dependence on the pore morphology and the dependence of the morphology on temperature.

The comparison between the weight fraction remaining versus time profiles that were obtained with the numerical solution and the measured response is shown in Fig. 6 . The numerical results are shown with solid lines and hollow data points and the measured results are shown with dashed lines and filled data points. In Fig. $6 \mathrm{a}$, the results at $700{ }^{\circ} \mathrm{C}$, $800{ }^{\circ} \mathrm{C}, 900{ }^{\circ} \mathrm{C}$ and $950{ }^{\circ} \mathrm{C}$ are shown using squares, circles, triangles and diamonds, respectively. In Fig. $6 \mathrm{~b}$, the results at $600{ }^{\circ} \mathrm{C}$ and $700{ }^{\circ} \mathrm{C}$ are shown using circles and squares, respectively.

Note the close agreement between the measured and predicted profiles at $800{ }^{\circ} \mathrm{C}$, $900{ }^{\circ} \mathrm{C}$ and $950{ }^{\circ} \mathrm{C}$, whereas the agreement at $600{ }^{\circ} \mathrm{C}$ and $700{ }^{\circ} \mathrm{C}$ is not as close. The discrepancy lies in the fact that the measured weight loss curves are sigmoidal and, as discussed previously, the numerical method neglects to account for the initial low rate of carbon oxidation. We note, however, that there is close agreement between the slopes of the measured and predicted weight fraction curves at $600{ }^{\circ} \mathrm{C}$ and $700{ }^{\circ} \mathrm{C}$ for all points below a weight fraction remaining of 0.9 . 
In Fig. 7, the spatial distribution of the carbon volume fraction is plotted at 1, 2 and $3 \mathrm{hrs}$ for the numerical simulation at $700{ }^{\circ} \mathrm{C}$ and, in Fig. 8 , the spatial distribution of the carbon volume fraction is plotted for 10,20 and $30 \mathrm{hrs}$ for the numerical simulation at $950{ }^{\circ} \mathrm{C}$. In addition to the fact that the rate of oxidation is much slower at $950^{\circ} \mathrm{C}$, we note the distinct difference between the carbon volume fraction distribution shown in Fig. 8, and that which is shown in Fig. 7. At $700{ }^{\circ} \mathrm{C}$, the carbon volume fraction distribution is much more uniform. The carbon volume fraction profile at $950{ }^{\circ} \mathrm{C}$, however, becomes more and more slender with time. Carbon oxidation occurs at the outer edge of the specimen with little or no oxidation in the interior. As time progresses, the delineation between the oxidized and non-oxidized zones marches inward from the outer surface, the so-called shrinking core oxidation behavior [4]. It is the variation of the areal porosity with temperature that is responsible for the distinct difference in the oxidation patterns shown in Figs. 7 and 8 , since areal porosity regulates the availability of oxidant in the interior of the specimen.

The difference between the oxidation behavior in the two temperature regimes, which is illustrated in Figs. 7 and 8 has been observed by Verrilli and Calomino [15] in constant-load rupture test specimens tested in a partial-oxygen environment at $800{ }^{\circ} \mathrm{C}$ and $1200^{\circ} \mathrm{C}$ as well as by Halbig [16]. They observed a shrinking core oxidation pattern at the higher temperature and a more uniform oxidation pattern in the specimens tested at the lower temperature. The temperature at which the oxidation behavior transitions from a somewhat uniform pattern to a shrinking core pattern is somewhere between $700{ }^{\circ} \mathrm{C}$ and $950{ }^{\circ} \mathrm{C}$ in the TGA experiment, whereas this transition temperature is between $800{ }^{\circ} \mathrm{C}$ 
and $1200{ }^{\circ} \mathrm{C}$ in the rupture tests. The fact that the transition temperature is higher in rupture test specimens than in TGA test specimens makes physical sense when we consider that the rupture test specimens are loaded in tension and since a tension stress will tend to increase the areal porosity by increasing the volumetric porosity and by lessening the tortuosity.

\section{CONCLUDING REMARKS}

A mathematical theory and an accompanying numerical scheme have been developed for predicting the oxidation behavior of $\mathrm{C} / \mathrm{SiC}$ composite structures. The theory is derived from the mechanics of the flow of ideal gases through a porous solid. The result of the theoretical formulation is a set of two coupled nonlinear differential equations written in terms of the oxidant and oxide partial pressures. The numerical method is based upon the solution of the two nonlinear differential equations using the Bubnov-Galerkin finite element method. The nonlinear differential equations are linearized within each time step and solved over the time domain in a piecewise linear manner. This is achieved by continuously updating the system stiffness matrix and system force vector based on the values of the solution variables determined in the previous time step. The end result is a numerical scheme capable of determining the variation of the local carbon oxidation rates as a function of space and time for any arbitrary $\mathrm{C} / \mathrm{SiC}$ composite structure. 
In the application section, the numerical method was applied to simulate the oxidation and weight loss behavior of $\mathrm{C} / \mathrm{SiC}$ composite specimens in the thermogravimetric experiments performed by Halbig [4]. To this end, it was necessary to determine the variation of the areal porosity and the surface area fraction with temperature. The numerical method was used to deduce the variation of these two material parameters with temperature.

The numerical simulation method was successful in reproducing the carbon volume fraction spatial distribution patterns which have been observed in $\mathrm{C} / \mathrm{SiC}$ stress rupture specimens, distribution patterns which are characteristic of the test temperature. Temperature was shown to have a profound influence on the oxidation behavior, both by its direct influence on the chemical kinetics and by its effect on the areal porosity and surface area fractions. 
Appendix: Expressions for the Elements of the Capacitance and Stiffness Matrices and Force Vector in Equation (12)

$$
\begin{aligned}
& (\boldsymbol{C O X})_{i j}=\phi \frac{M_{o x}}{R \hat{T}} \int_{D^{e}} N_{i} N_{j} d D^{e} \\
& (\boldsymbol{C C O})_{i j}=\phi \frac{M_{c o}}{R \hat{T}} \int_{D^{e}} N_{i} N_{j} d D^{e} \\
& (\boldsymbol{K} \boldsymbol{O X})_{i j}=\frac{\hat{\rho}_{o x}^{p}}{\hat{\mu}_{g}} \int_{D^{e}} \nabla N_{i} \cdot \mathbf{k} \cdot \nabla N_{j} d D^{e}+D_{A B} \frac{M_{o x}}{R \hat{T}} \frac{\hat{p}_{c o}}{\hat{p}} \int_{D^{e}} \nabla N_{i} \cdot \boldsymbol{\varphi}^{A} \cdot \nabla N_{j} d D^{e} \\
& (\boldsymbol{K X C})_{i j}=\frac{\hat{\rho}_{o x}^{p}}{\hat{\mu}_{g}} \int_{D^{e}} \nabla N_{i} \cdot \mathbf{k} \cdot \nabla N_{j} d D^{e}-D_{A B} \frac{M_{o x}}{R \hat{T}} \frac{\hat{p}_{o x}}{\hat{p}} \int_{D^{e}} \nabla N_{i} \cdot \boldsymbol{\varphi}^{A} \cdot \nabla N_{j} d D^{e} \\
& (\boldsymbol{K C X})_{i j}=\frac{\hat{\rho}_{c o}^{p}}{\hat{\mu}_{g}} \int_{D^{e}} \nabla N_{i} \cdot \mathbf{k} \cdot \nabla N_{j} d D^{e}-D_{A B} \frac{M_{c o}}{R \hat{T}} \frac{\hat{p}_{c o}}{\hat{p}} \int_{D^{e}} \nabla N_{i} \cdot \boldsymbol{\varphi}^{A} \cdot \nabla N_{j} d D^{e} \\
& (\boldsymbol{K C O})_{i j}=\frac{\hat{\rho}_{c o}^{p}}{\hat{\mu}_{g}} \int_{D^{e}} \nabla N_{i} \cdot \mathbf{k} \cdot \nabla N_{j} d D^{e}+D_{A B} \frac{M_{c o}}{R \hat{T}} \frac{\hat{p}_{o x}}{\hat{p}} \int_{D^{e}} \nabla N_{i} \cdot \boldsymbol{\varphi}^{A} \cdot \nabla N_{j} d D^{e} \\
& (\boldsymbol{F O X})_{i}=\lambda_{1} \frac{M_{o x}}{M_{c}} \int_{D^{e}} N_{i} N_{j}\left(\Re_{c f_{j}}+\Re_{p c}\right) d D^{e}+\phi \frac{\hat{\rho}_{o x}^{p}}{\hat{T}} \frac{d T}{d t} \int_{D^{e}} N_{i} d D^{e} \\
& -\oint N_{i} J_{o x} \cdot \boldsymbol{n} d \Gamma^{e} \\
& \Gamma^{e} \\
& (\boldsymbol{F C O})_{i}=-\lambda_{2} \frac{M_{c o}}{M_{c}} \int_{D^{e}} N_{i} N_{j}\left(\Re_{c f_{j}}+\Re_{p c j}\right) d D^{e}+\phi \frac{\hat{\rho}_{c o}^{p}}{\hat{T}} \frac{d T}{d t} \int_{D^{e}} N_{i} d D^{e} \\
& -\oint_{\Gamma^{e}} N_{i} \boldsymbol{J}_{c o} \cdot \boldsymbol{n} d \Gamma^{e}
\end{aligned}
$$




\section{References}

[1] Lamouroux F, Bourrat X, Nasalain R, Sevely J. Structure/oxidation behavior relationship in the carbonaceous constituents of $2 \mathrm{D} \mathrm{C} / \mathrm{PyC} / \mathrm{SiC}$ composites. Carbon 1993; 31 (8): 1273-1288.

[2] Medford JE. Prediction of oxidation performance of reinforced carbon-carbon material for space shuttle leading edges. $10^{\text {th }}$ AIAA Thermophysics Conference Denver (Colorado, USA): AIAA Paper No. 75-730, 1975.

[3] Eckel AJ, Cawley JD, Parthasarathy TA. Oxidation kinetics of a continuous carbon phase in a nonreactive matrix. Journal of the American Ceramics Society 1995; 78 (4): 972-980.

[4] Halbig MC. The Oxidation kinetics of continuous carbon fibers in a cracked ceramic matrix composite. NASA/TM-2001-210520. 2001.

[5] Darcy H. Les Fontaines Publiques de la ville de Dijon. Dalmont, Paris: 1856.

[6] Bird, Stewart, Lightfoot. Transport Phenomena. New York, NY: Wiley. 1960.

[7] Bacos MP, Dorvaux JM, Lavigne O, Talandier J. C/C Composite oxidation model -

III. Physical basis, limitations and applications. Carbon 2000; 38 (1): 105-117.

[8] Gulbransen EA, Andrew KF, Brassart FA. The Oxidation of graphite at temperatures of $600{ }^{\circ} \mathrm{C}$ to $1500{ }^{\circ} \mathrm{C}$ and at pressures of 2 to 76 torr of oxygen. Journal of the Electrochemical Society $1963 ; 110$ (6): 476-483.

[9] Bathe KJ. Finite Element Procedures in Engineering Analysis. Englewood Cliffs, NJ: Prentice-Hall Inc. 1982.

[10] Burnett DS. Finite Element Analysis: From Concepts to Applications. Reading, MA: Addison-Wesley Publishing Company. 1987.

[11] Opila E. Oxidation kinetics of chemically vapor-deposited silicon carbide in wet oxygen. Journal of the American Ceramics Society 1994; 77 (3): 730-736.

[12] Halbig MC. Carbon oxidation studies and the evaluation of oxidation inhibited $\mathrm{C} / \mathrm{SiC}$ composites. $28^{\text {th }}$ annual conference on composites, materials and structures Cocoa Beach (Florida, USA): United States Advanced Ceramics Association, 2004.

[13] Ismail IMK. On the reactivity, structure and porosity of carbon fibers and fabrics. Carbon 1991; 29: 777-792.

[14] Walker PL Jr., Rusinko F Jr., Austin LG. Gas reactions of carbon. In Eley, DD, Selwood PW, Weisz PB editors, Advances in Catalysis and Related Subjects, Vol. 11, Academic Press, Inc., New York and London: 1959: 133-221.

[15] Verrilli M, Calomino A. Temperature dependence on the strength and stress rupture behavior of a carbon fiber-reinforced silicon carbide matrix $(\mathrm{C} / \mathrm{SiC})$ composite. Ceramic Engineering and Science Proceedings 2003; 24 (4): 443-449.

[16] Halbig MC. The influence of temperature, stress and environment on the oxidation and life of $\mathrm{C} / \mathrm{SiC}$ composites. Ceramic Engineering and Science Proceedings 2002; 23 (3): 419-426. 


\section{Figure Captions}

Fig. 1. Optical Microscopy of 2-D C/SiC Composite (Courtesy of Southern Research Institute, Birmingham, AL).

Fig. 2. Flow Chart Showing the Steps Involved in the Numerical Solution Routine.

Fig. 3. Weight Loss Versus Time Profiles for Various Temperatures Measured in the TGA Experiment (Halbig [4]).

Fig. 4. Finite Element Mesh and Boundary and Initial Conditions Used for the Numerical Simulation of the TGA Experiment.

Fig. 5. Plot of the Proposed Dependence of Areal Porosity and Exposed Surface Area Fraction with Temperature.

Fig. 6. Comparsion of Measured and Predicted Weight Fraction Remaining Versus Time Profiles for $600,700,900$ and $950{ }^{\circ} \mathrm{C}$.

Fig. 7. Predicted Spatial Distribution of Carbon Volume Fraction at 1,2 and 3 hours at $700^{\circ} \mathrm{C}$.

Fig. 8. Predicted Spatial Distribution of Carbon Volume Fraction at 10, 20 and 30 hours at $950{ }^{\circ} \mathrm{C}$. 


\section{Table 1. List of Material Constant Values Used for TGA Oxidation Simulation}

\begin{tabular}{|c|c|}
\hline Material Constant & Value \\
\hline$\widetilde{\rho}_{c}(\mathrm{~g} / \mathrm{cc})$ & 1.74 \\
\hline$k_{o}^{c f}=k_{o}^{p c}\left(\mathrm{sec}^{-1}\right)$ & 6452.35 \\
\hline$n_{c f}=n_{p c}$ & 1 \\
\hline$E_{a}^{c f}=E_{a}^{p c}(\mathrm{~kJ} / \mathrm{mole})$ & 118.3 \\
\hline$v_{c f}^{o}+v_{p c}^{o}$ & 0.5 \\
\hline$\phi$ & 0.1 \\
\hline
\end{tabular}




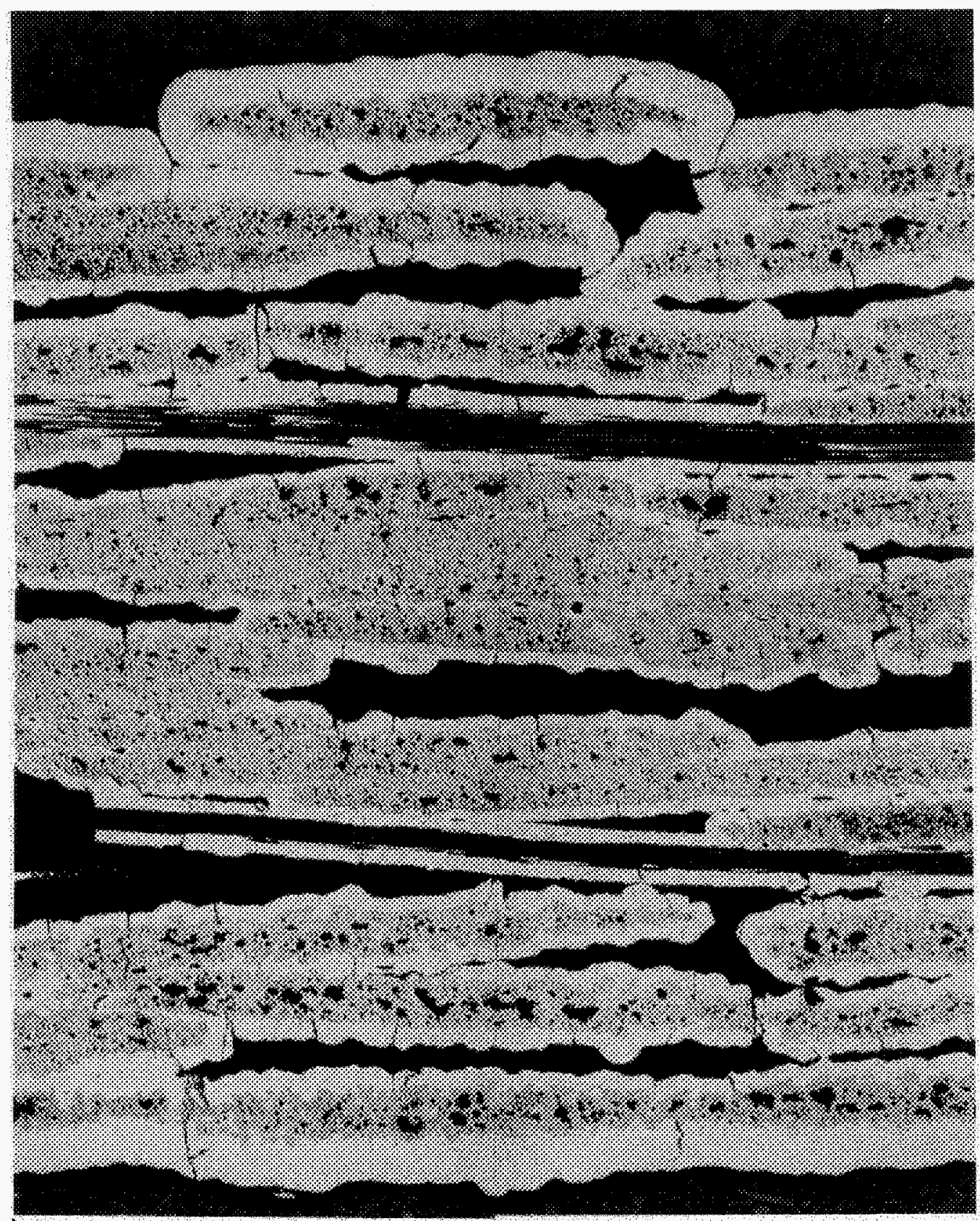

Fig. 1. Optical Microscopy of 2-D C/SiC Composite (Courtesy of Southern Research Institute, Birmingham, AL). 
Initlalize the partial pressures and partial densities for oxygen and oxides and initialize the material parameters.

Form the element capacitance matrix $[C]$ for each finite element using equation (12) and the equations in the Appendix.

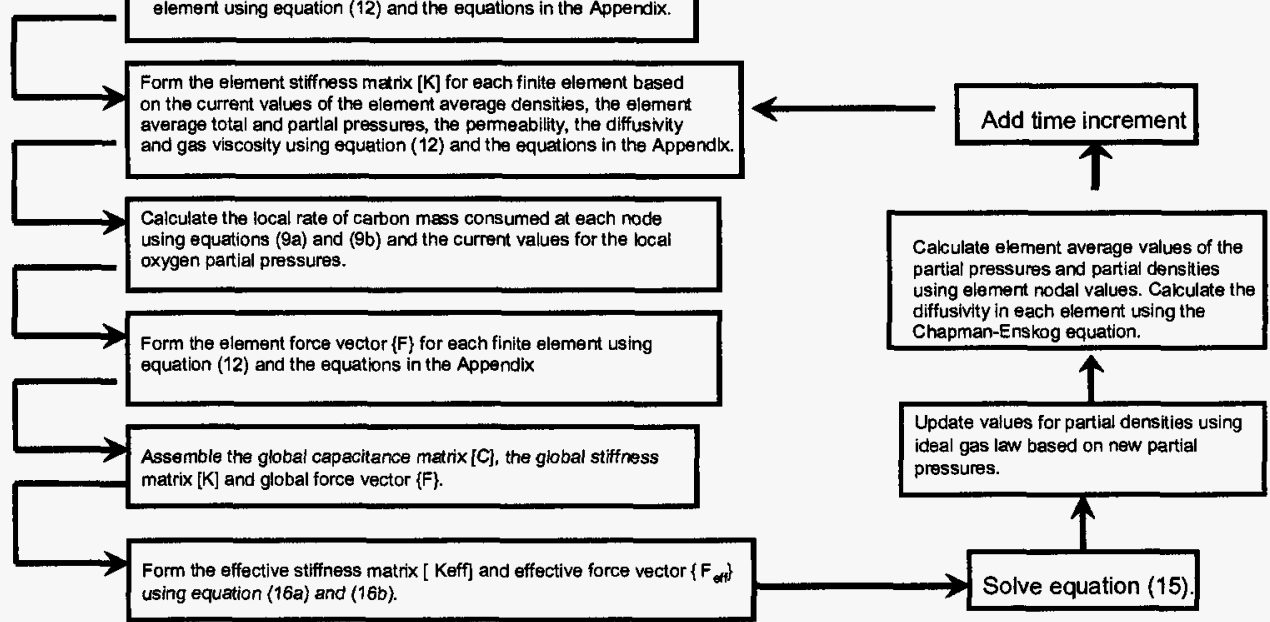

Fig. 2. Flow Chart Showing the Steps Involved in the Numerical Solution Routine. 


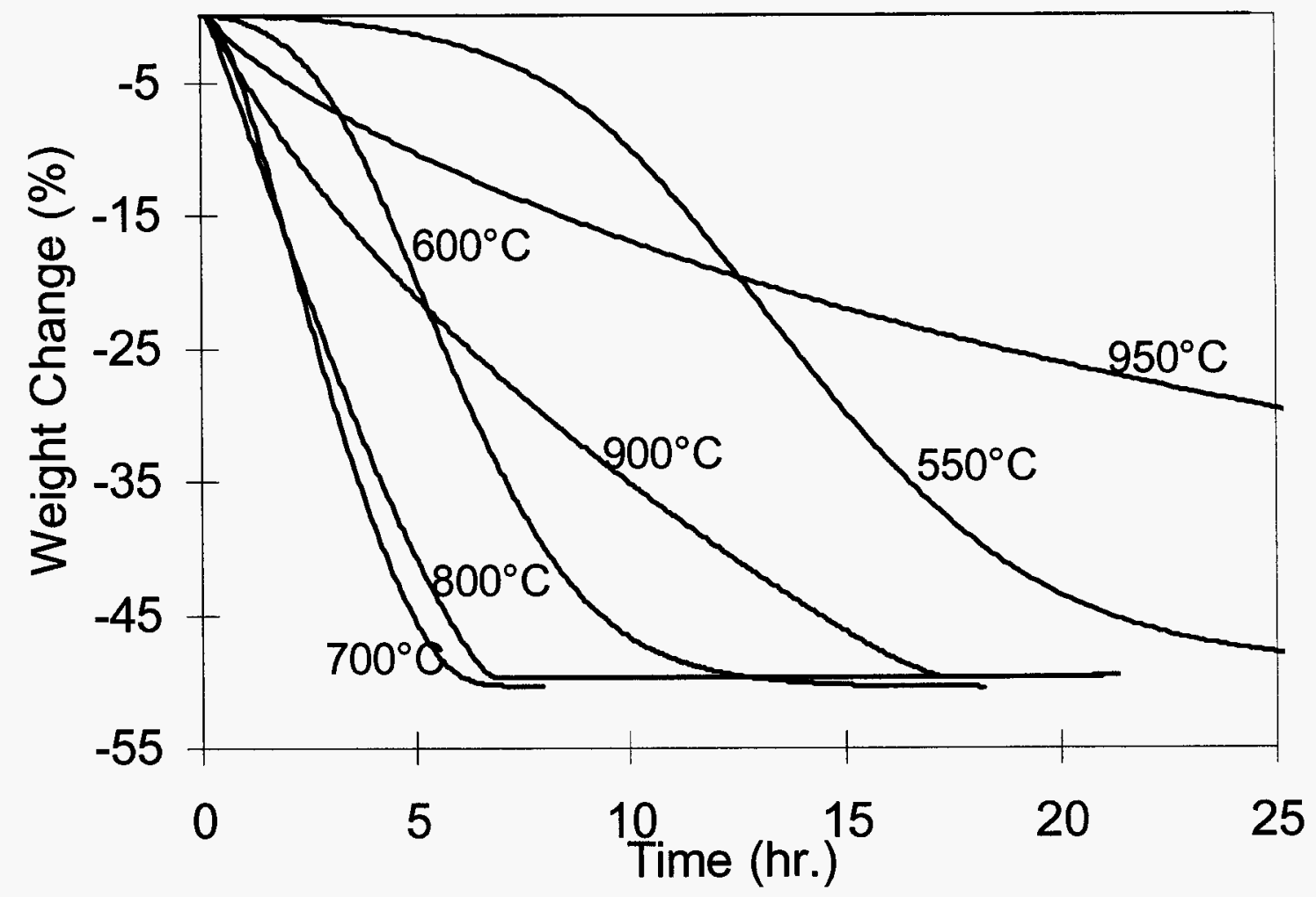

Fig. 3. Weight Loss Versus Time Profiles for Various Temperatures Measured in the TGA Experiment (Halbig [4]). 


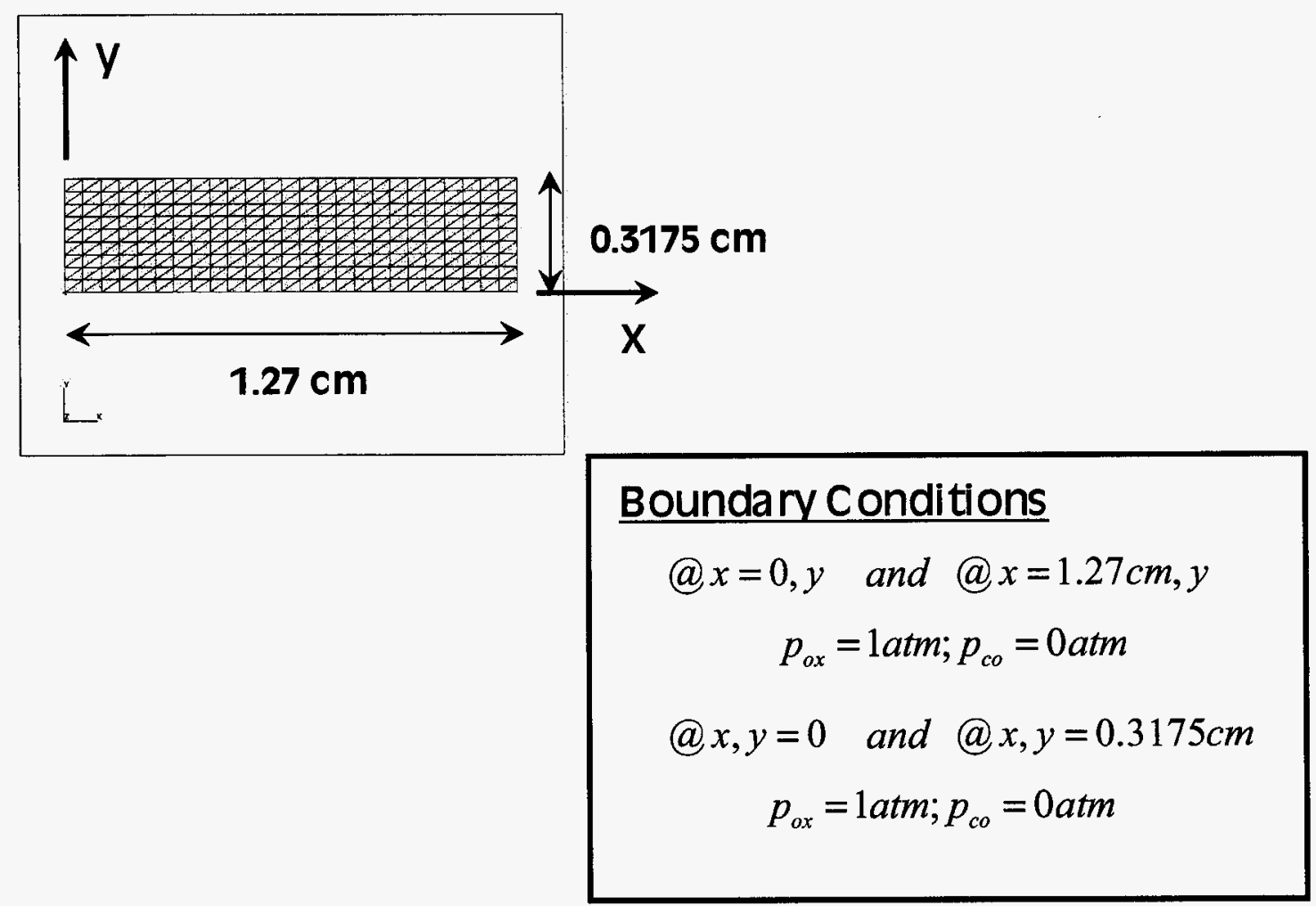

Fig. 4. Finite Element Mesh and Boundary and Initial Conditions Used for the Numerical Simulation of the TGA Experiment. 


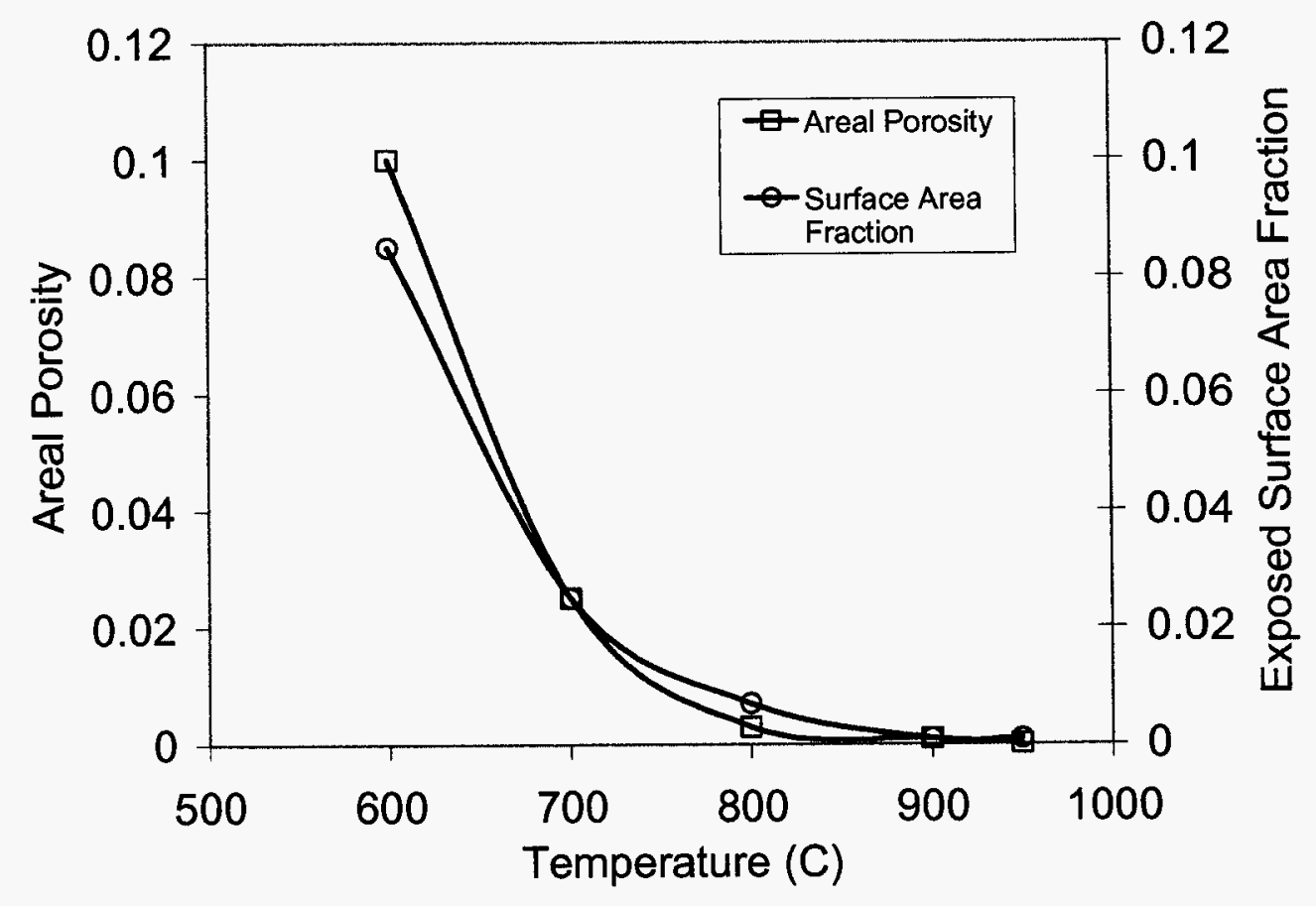

Fig. 5. Plot of the Proposed Dependence of Areal Porosity and Exposed Surface Area Fraction with Temperature. 


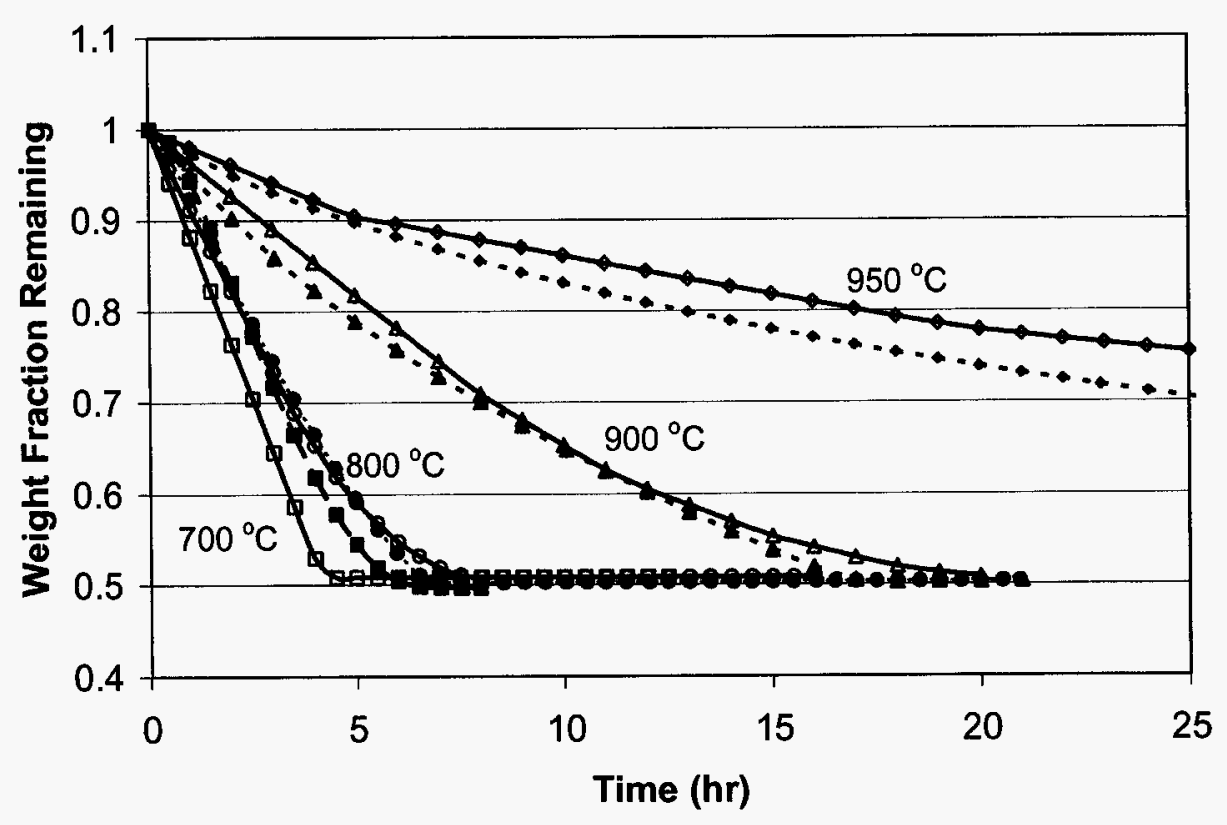

a. Results at $700{ }^{\circ} \mathrm{C}, 800{ }^{\circ} \mathrm{C}, 900{ }^{\circ} \mathrm{C}$ and $950{ }^{\circ} \mathrm{C}$.

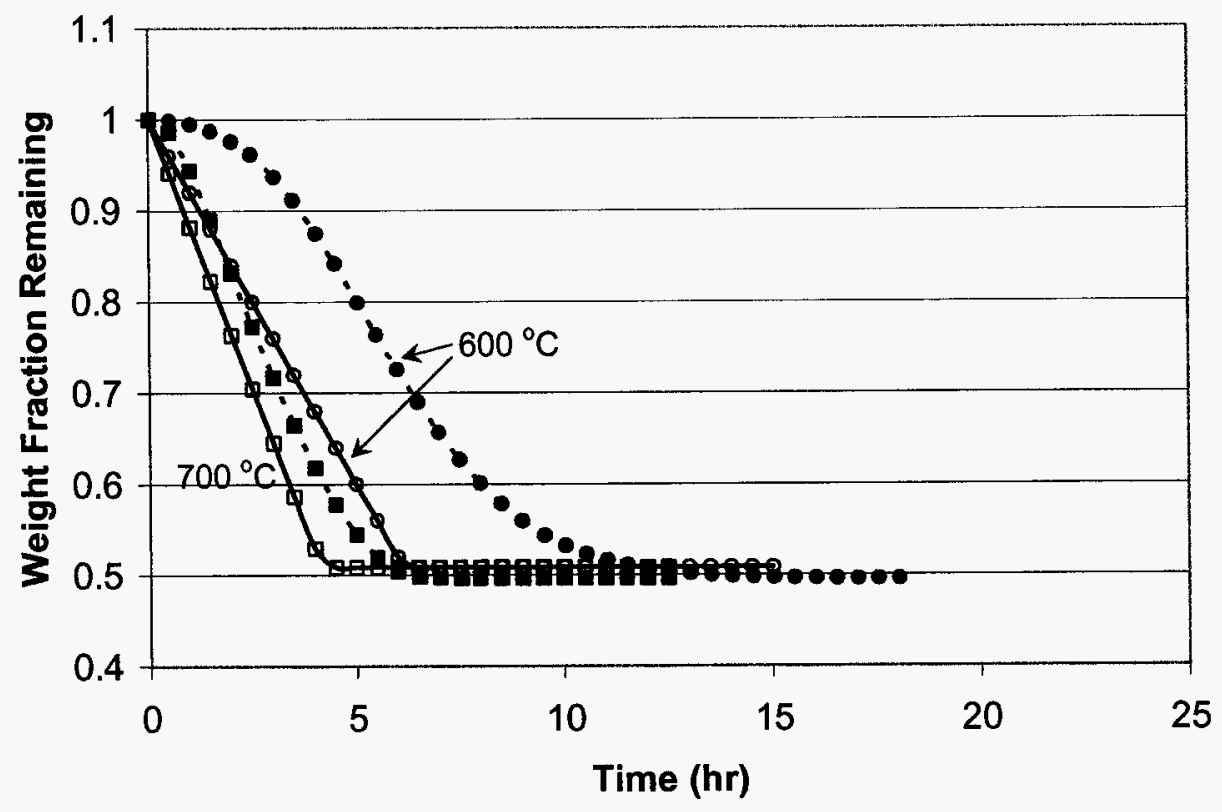

b. Results at $600^{\circ} \mathrm{C}$ and $700^{\circ} \mathrm{C}$.

Fig. 6. Comparsion of Measured and Predicted Weight Fraction Remaining Versus Time Profiles for $600,700,800,900$ and $950^{\circ} \mathrm{C}$. 


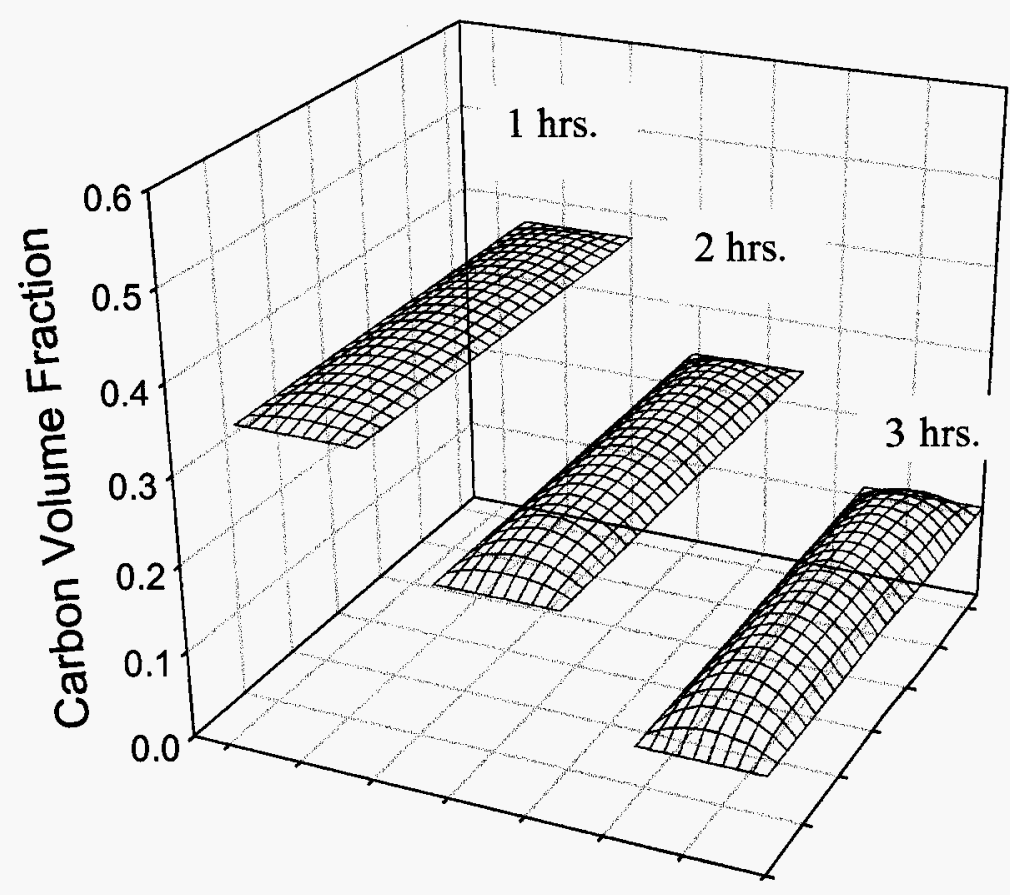

Fig. 7. Predicted Spatial Distribution of Carbon Volume Fraction at 1,2 and 3 hours at $700{ }^{\circ} \mathrm{C}$.

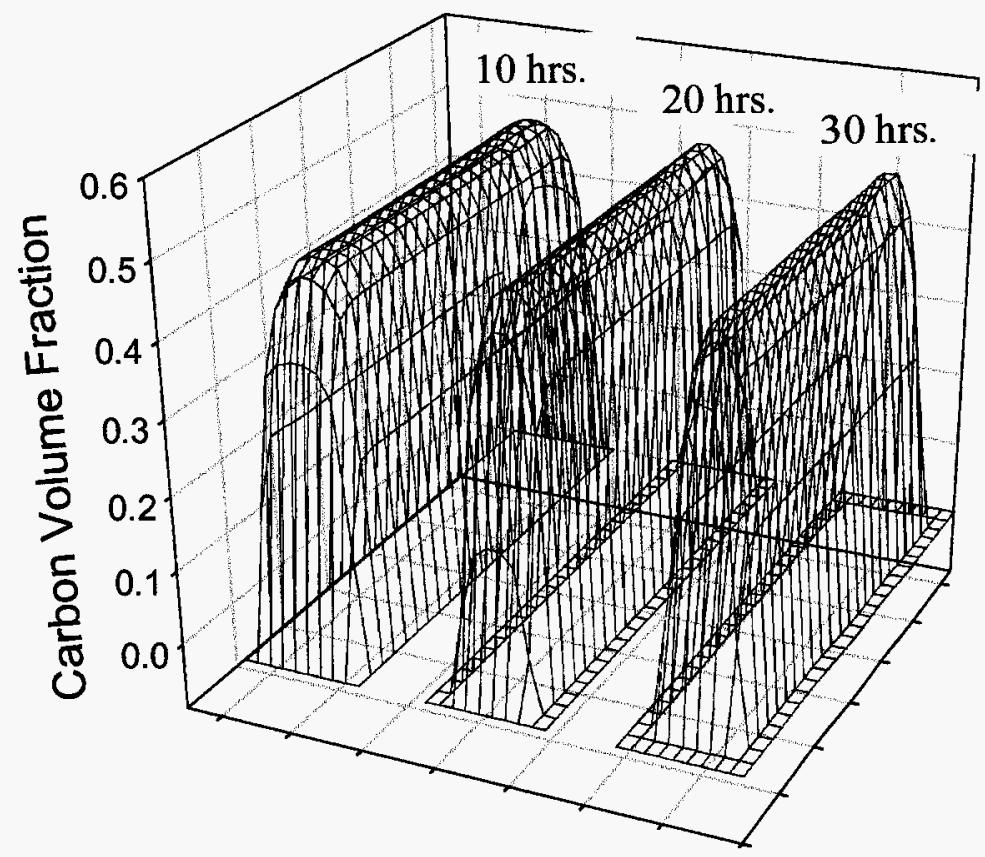

Fig. 8. Predicted Spatial Distribution of Carbon Volume Fraction at 10,20 and 30 hours at $950^{\circ} \mathrm{C}$. 